of revisionism, but at least the fundamentals appear to be in place.

Andrew Jephcoat and Keith Refson are in the

Department of Earth Sciences, University of Oxford,

Oxford OX1 3PR, UK.

e-mails: andrew@earth.ox.ac.uk

keith@earth.ox.ac.uk

Cytoskeleton

\section{Evolution in bacteria}

\author{
Harold P. Erickson
}

Actin is a major component of the cytoskeleton in yeast, plant and animal cells, but when did it evolve? The discovery of a bacterial protein that forms actin-like filaments suggests an answer.

A ll eukaryotic cells, from yeast to plants to animals, have an internal framework called the cytoskeleton. The three types of strut that make up this framework microtubules, actin filaments and intermediate filaments - not only provide cells with mechanical support, but also serve as tracks for motor molecules to move along. Bacteria were long thought to lack cytoskeletal filaments, suggesting that the cytoskeleton might have evolved after the first primitive eukaryotic cell developed from its bacterial origins.

Over the past few years, however, microtubules have been traced back to the bacterial protein FtsZ, which forms filaments that are involved in cell division. The origin of actin has been more obscure (and even less is known about the evolution of intermediate filaments). Now, work by van den Ent and colleagues $^{1}$ (on page 39 of this issue), along with a paper by Jones and co-workers ${ }^{2}$, shows that the protein MreB forms an actin-like cytoskeleton in bacteria, providing a strong argument that the actin cytoskeleton had largely evolved before eukaryotes developed.

Several bacterial proteins are related to actin, as judged by similarities in their amino-acid sequences. But although this 'homology' shows that the proteins evolved from a common ancestor, it says nothing about their functions. In fact, these bacterial relatives have widely different functions, most of which are unrelated to the cytoskeleton. Hexokinase is an enzyme; DnaK is a 'chaperone' protein that helps other proteins to fold correctly; and FtsA functions in cell division, although perhaps not as a cytoskeletal protein. MreB, another bacterial relative of actin, is somehow involved in controlling the shape of Escherichia coli cells ${ }^{3}$, but its precise function has been uncertain. If one of these actin homologues is a direct evolutionary precursor of actin, it might be expected to assemble into filaments. The new studies ${ }^{1,2}$ show that MreB does just that.

Both groups ${ }^{1,2}$ noted that, in terms of sequence, MreB is closer to actin than are the other bacterial homologues. Jones et al. ${ }^{2}$
1. Creager, K. C. J. Geophys. Res. 104, 23127-23139 (1999).

3. Tromp, J. Annu. Rev. Earth Planet. Sci. 29, 47-69 (2001).

4. Steinle-Neumann, G., Stixrude, L. Cohen, R. E. \& Gülseren, O. Nature 413, 57-60 (2001)

5. Buffett, B. A. \& Wenk, H.-R. Nature 413, 60-63 (2001).

6. Stixrude, L. \& Cohen, R. E. Science 267, 1972-1975 (1995).

7. Alfe, D., Price, G. D. \& Gillan, M. J. Phys. Rev. B 64, 45123-45139 (2001).
2. Vidale, J. E. \& Earle, P. S. Nature 404, 273-275 (2000). used light microscopy to examine MreB and a closely related protein, $\mathrm{Mbl}$, in Bacillus subtilis cells. They found that these proteins form separate helical bands that spiral around inside the cell, just under the membrane. They went on to show that depletion of either protein causes defects in cell shape: MreB affects mainly the width, and Mbl the length, of the rod-shaped B. subtilis. Similarly, deletion of MreB in E. coli cells results in a marked switch from a rod shape to a sphere ${ }^{3}$.

To generalize these findings, Jones et al. scanned the numerous bacterial and archaeal genomes that have been sequenced. (Bacteria and archaea are the two groups of prokaryotes - organisms without membranebound nuclei.) They found that prokaryotes with a rod-like, filamentous or helical shape generally have one or more $m r e B$ genes, but spherical bacteria (cocci) have none. So the default shape for a bacterium seems to be a sphere; other types of shape are produced by an MreB-based cytoskeleton.

The spiral bands seen by light microscopy were suggestive of a cytoskeleton with a filamentous substructure, but this needed to be demonstrated. van den Ent et al. ${ }^{1}$ do exactly this, and more. They first show that the Thermotoga maritima MreB protein forms polymers in vitro. Electron microscopy revealed that the polymers are thin 'protofilaments' (consisting of a single row of MreB proteins assembled end-to-end) that line up side-byside to form pairs or larger parallel arrays. The spacing between MreB proteins along the protofilaments is $51 \AA$, close to the $55-\AA$ spacing between subunits in eukaryotic actin.

But there is one striking difference. In eukaryotes, two actin protofilaments coil around each other to form a helical actin filament, whereas the MreB protofilaments are straight. Although biologists interested in the cytoskeleton have always thought of the helical structure as fundamental to actin, van den Ent et al. ${ }^{1}$ present an intriguing argument that the natural shape of an isolated actin protofilament may in fact be straight, but that the two protofilaments in an actin filament are distorted into helices by the lateral contacts that hold them together.

The authors ${ }^{1}$ went on to crystallize MreB and determine its atomic structure. The structure confirms the similarity of MreB to actin, and these particular crystals also provide an amazing bonus: the MreB subunits are already assembled into protofilaments. The crystals thus reveal the structure, at atomic resolution, of the interface between subunits in the protofilaments. This interface has not been seen in crystals of actin, and has been deduced only by modelling. So the MreB structure provides the first detailed look at the interface that underlies actin-like filaments.

The helical bands seen by light microscopy ${ }^{2}$ probably represent a network or a parallel bundle of MreB protofilaments. But how many protofilaments are there across the width of each band? Jones et al. ${ }^{2}$ show that there are 8,000 molecules of MreB and 13,000 of $\mathrm{Mbl}$ in an average bacterium. Assuming that both of these proteins form protofilaments with the $51-\AA$ spacing observed by van den Ent et al. ${ }^{1}$, this is enough to assemble $41 \mu \mathrm{m}$ and $66 \mu \mathrm{m}$ of MreB and Mbl protofilaments, respectively. The bacteria are about $1 \mu \mathrm{m}$ in diameter, giving a circumference of $3 \mu \mathrm{m}$. So, as the helical bands are judged to make one or two turns, they would have a length of 3-6 $\mu \mathrm{m}$. What this means is that the bands seen by light microscopy might be about 10 protofilaments wide, which could provide significant mechanical rigidity.

It seems likely that the MreB-based cytoskeleton determines cell shape by generating a force against the membrane. How might it do this? In eukaryotes, a molecular motor known as myosin, which moves along actin tracks, can generate contractile force. But no bacterial motor proteins have been identified, so a similar mechanism seems unlikely to operate in bacteria. A force could instead be generated simply by the filaments' mechanical rigidity, which would prevent them from bending. A third mechanism of force generation in eukaryotes is based on filament assembly: as new subunits are added to the ends of filaments, they push against the membrane. This is the basis for protrusion of the leading edges of amoebae and amoeboid animal cells as they move across a substrate ${ }^{4}$. Perhaps this fundamental process of cell motility, as well as the actin cytoskeleton itself, evolved in bacteria before eukaryotes formed.

Harold P. Erickson is in the Department of Cell Biology, Duke University Medical Center, Durham,

North Carolina 27710, USA.

e-mail: h.erickson@cellbio.duke.edu

1. van den Ent, F., Amos, L. A. \& Löwe, J. Nature 413, 39-44 (2001).

2. Jones, L. J., Carballido-Lopez, R. \& Errington, J. Cell 104, 913-922 (2001).

3. Doi, M. et al. J. Bacteriol. 170, 4619-4624 (1988).

4. Pollard, T. D., Blanchoin, L. \& Mullins, R. D. Annu. Rev Biophys. Biomol. Struct. 29, 545-576 (2000). 